\title{
Localization of Sodium Channel Subtypes in Adult Rat Skeletal Muscle Using Channel-Specific Monoclonal Antibodies
}

\author{
Beatrice Haimovich, Donald L. Schotland, William E. Fieles, and Robert L. Barchi \\ The David Mahoney Institute of Neurological Sciences, the Henry Watts Jr. Neuromuscular Disease Research Center, \\ and the Department of Neurology, University of Pennsylvania School of Medicine, Philadelphia, Pennsylvania 19104
}

Five monoclonal antibodies specific for the $260 \mathrm{kDa}$ subunit of the rat skeletal muscle sodium channel were used to probe the distribution of this channel in adult muscle. All the antibodies reacted with the surface membrane of fast- and slow-twitch fibers in the rat anterior tibial and soleus muscles. Immunoreactivity was also present in the endplate region; this was significantly more intense than that in the surrounding extrajunctional membrane. At the electron microscopic level, this junctional immunoreactivity could be traced uniformly throughout the secondary folds of the postsynaptic membrane.

Three of the monoclonal antibodies (A/B2, F/E4, and I/E3) exhibited an additional distinct immunoreactivity pattern, staining the interior of selected fibers in the anterior tibial muscle that were subsequently identified as slow-twitch fibers. An identical reactivity pattern was observed with most of the soleus muscle fibers. In longitudinal sections of slow fibers examined at the light microscopic level, transversely oriented, regularly spaced doublets of fluorescence were localized at the junction of the $A$ and $I$ bands in each sarcomere. In permeabilized slow fibers exposed to A/B2 and examined at the electron microscopic level, internal reactivity was associated exclusively with the membranes of the T-tubular system. A/B2 also strongly stained a transversely oriented pattern within cardiac muscle fibers exhibiting the characteristics of the T-tubular system in that tissue.

We conclude that at least 3 subpopulations of sodium channels are present in adult skeletal muscle: those in the sarcolemma of fast and slow fibers, those in slow-twitch fiber T-tubular membranes, and those in the T-tubular system of fast fibers. The channels in the slow fiber T-system apparently share common epitopes with those in the T-system of cardiac fibers.

Voltage-sensitive sodium channels that control the transient inward sodium current during an action potential are characteristic elements of most nerve and muscle cell membranes. In skeletal muscle, sodium channels are usually found in 2 membrane systems: the surface membrane or sarcolemma, which propagates the action potential along the length of the fiber, and

Received Nov. 25, 1986; revised Mar. 13, 1987; accepted Mar. 25, 1987.

We wish to thank Drs. N. A. Rubinstein and A. F. Kelly for their gift of actomyosin samples used in our control experiments, and Dr. Z. W. Hall for providing us with labeled $\alpha$-bungarotoxin. This work was supported in part by NIH Grants NS-08075 and NS-18013, and by a grant from the Muscular Dystrophy Association.

Correspondence should be addressed to Dr. B. Haimovich, Mahoney Institute of Neurological Sciences, University of Pennsylvania School of Medicine, Philadelphia, PA 19104.

Copyright (C) 1987 Society for Neuroscience $0270-6474 / 87 / 092957-10 \$ 02.00 / 0$ the transverse tubular (T-tubular) system, whose membranes conduct this signal radially into the fiber interior (Constantin and Taylor, 1971).

Although sodium channels from disparate sources are very similar in their physical and biochemical properties (reviewed by Barchi, 1984; $\Lambda$ gnew, 1984; Catterall, 1984), studies with neurotoxins that affect channel function suggest that subtle differences may exist between related sodium channels of different species, as well as between channels from different tissues of the same animal. Sodium channels in adult skeletal muscle (Ritchie and Rogart, 1977; Chicheportiche et al., 1980) are blocked by TTX at concentrations 100 -fold lower than those needed to inhibit this channel in cardiac muscle (Baer et al., 1976; Cohen et al., 1981) or in skeletal muscle after denervation (Harris and Thesleff, 1971; Pappone, 1980). Some polypeptide neurotoxins seem to discriminate between sodium channels in muscle and those in brain or nerve (Cruz et al., 1985; Moczydlowski et al., 1986).

Significant differences may even exist between sodium channels in the same tissues. In physiological experiments, 2 scorpion polypeptide neurotoxins blocked currents associated with the sarcolemmal sodium channel but not those mediated by the T-tubular channels (Jaimovich et al., 1982; Barhanin et al., 1984). The recent cloning of 3 closely related sodium channel messages expressed in adult rat brain lends further support to this concept (Noda et al., 1986).

At the present time, information concerning the heterogeneity of sodium channels is based primarily on neurotoxin binding studies and data are not available on the localization of these subtypes in adult skeletal muscle. In this study, we used monoclonal antibodies to the sodium channel $\alpha$-subunit to study the distribution of sodium channel subtypes in adult skeletal muscle using immunocytochemical techniques. Our results indicate that at least 3 subtypes of sodium channel can be differentiated in innervated adult muscle, segregated in part by their membrane distribution and in part by muscle fiber type.

\section{Materials and Methods}

The monoclonal antibodies used in this study were generated against rat skeletal muscle sodium channel as previously described (Casadei et al., 1984). Additional aspects of their characterization have been reported elsewhere (Casadei and Barchi, 1987). Supernatant and ascites of a hybridoma cell line producing the monoclonal immunoglobulin P3/X63-Ag8 were used as controls.

Purified contractile proteins. Actomyosin proteins and purified rabbit actin, troponin, and tropomyosin were kindly provided by Drs. N. A. Rubinstein and A. M. Kelly (University of Pennsylvania).

Immunocytochemistry. Frozen muscle sections $(4 \mu \mathrm{m})$ were prepared from fresh tissue samples and transferred to glass coverslips. Sections were first incubated for 30 min with $10 \%$ normal goat serum in PBS 

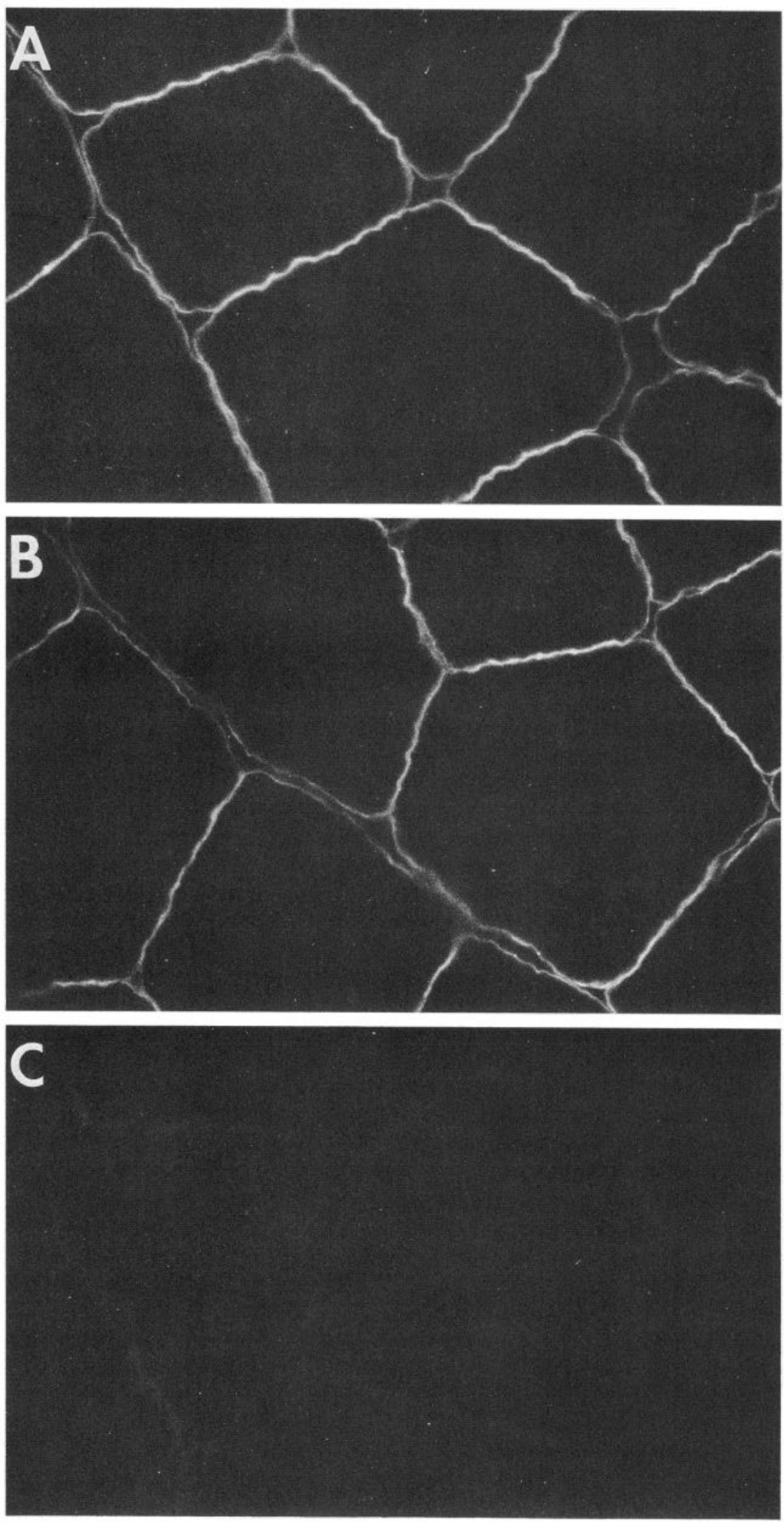

Figure 1. Monoclonal antibodies specific for the rat skeletal muscle sodium channel stained surface membranes of rat anterior tibial muscle in frozen cross sections. Unfixed sections were exposed to each monoclonal antibody, and bound antibody was subsequently localized with rhodamine-conjugated secondary antibody. Reactivity with 2 representative sodium channel antibodies is shown; micrographs are of muscle exposed to 1:50 dilution of monoclonal LD3 $(A)$, monoclonal AB2 $(B)$, or the control immunoglobulin Ag-8 (C). Original magnification, $\times 600$.

$(100 \mathrm{~mm} \mathrm{NaCl}, 50 \mathrm{~mm} \mathrm{KP}, \mathrm{pH} 7.4)$ to reduce nonspecific protein binding. Sections were then incubated for $30 \mathrm{~min}$ with the monoclonal antibodies (diluted 1:50 in 10\% normal goat serum), washed for $15 \mathrm{~min}$ in PBS, and incubated for another $30 \mathrm{~min}$ with rhodamine-conjugated goat anti-mouse secondary antibody (diluted 1:50 in 10\% normal goat serum) (Cappel Lab.), preabsorbed against rat liver powder as previously described (Haimovich et al., 1984). At the end of the incubation the sections were washed again as above.

In preparations counterstained with rhodamine-bungarotoxin, fluorescein-conjugated secondary antibody (Cappel Lab.) was used for an- 

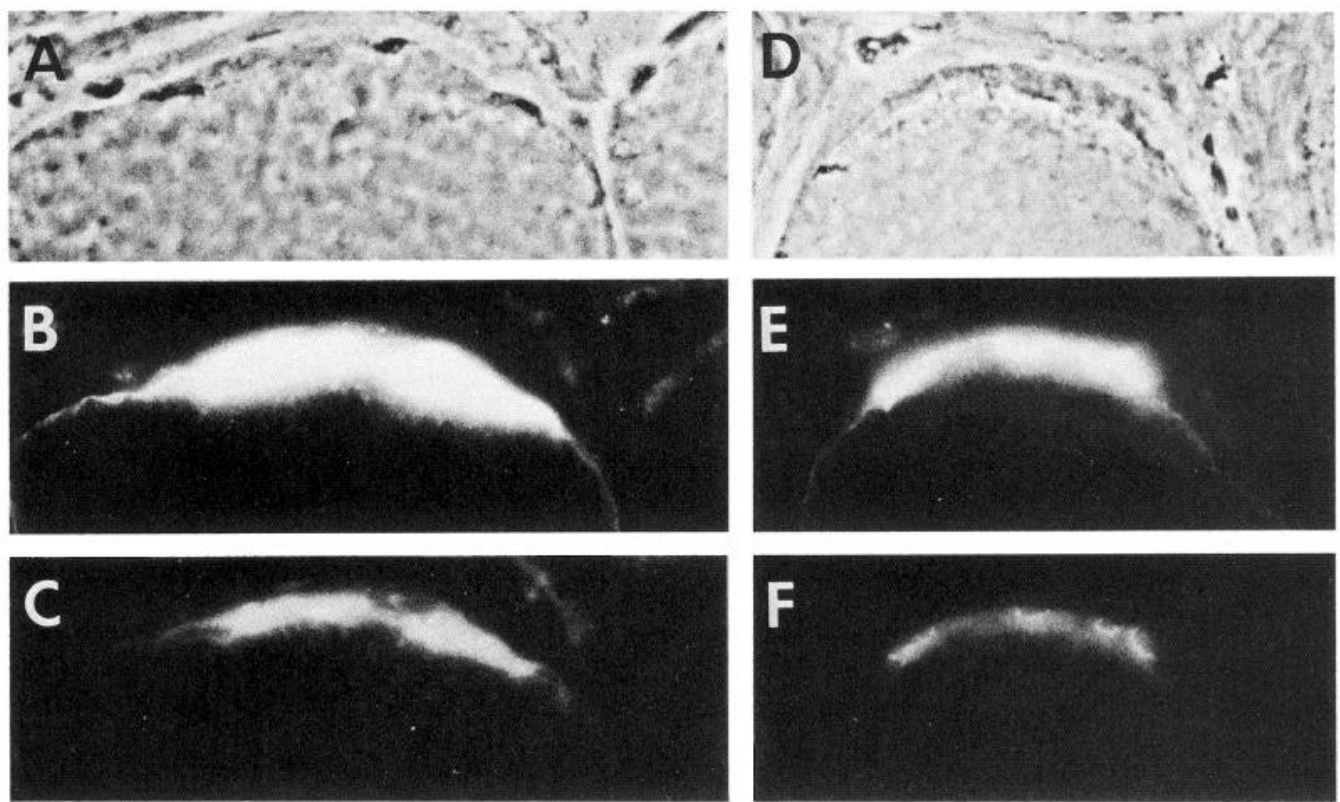

Figure 2. Antibodies to the sodium channel heavily label the endplate regions of skeletal muscle. In this figure, rat anterior tibial muscle sections were incubated with 1:50 dilution of monoclonal antibody $\mathrm{AB} 2$ and then exposed to fluorescein-conjugated secondary antibody and rhodamineconjugated $\alpha$-bungarotoxin. Areas of intense reactivity with AB2 coincided with regions labeled with $\alpha$-bungarotoxin. $A$ and $D$, Phase-contrast micrographs of representative fields containing endplates. $B$ and $E$, Reactivity of the monoclonal antibody AB2 in the same fields detected with fluorescein-conjugated goat anti-mouse immunoglobulin. $C$ and $F$, Location of post-junctional membrane in these fields with rhodamine-conjugated $\alpha$-bungarotoxin. Original magnification, $\times 600$.

tibody visualization. Primary antibody and rhodamine-bungarotoxin $(2 \mu \mathrm{g} / \mathrm{ml})$ were added concomitantly to the muscle sections. Rhodamine $\alpha$-bungarotoxin was kindly provided by Dr. Z. W. Hall (University of California).

The sections were mounted in glycerol gel and examined with a Zeiss II photomicroscope equipped with epifluorescence optics.

Histochemistry. Frozen muscle sections, $6 \mu \mathrm{m}$ thick, were stained for myofibrillar ATPase as previously described (Padykula and Herman, 1955; Brook and Kaiser, 1969).

Electron microscopy. Thin bundles of adult anterior tibial and soleus muscles were fixed in a stretched position on paraffin for $1 \mathrm{hr}$ in $1 \%$ paraformaldehyde $\left(\mathrm{pH} \mathrm{7.4)}\right.$ at $4^{\circ} \mathrm{C}$. For half of the bundles, saponin $(0.05 \%)$ was added to the fixation medium and to each of the solutions used for processing subsequently; saponin-treated and control muscle were otherwise treated identically. The outer portions of the muscle were cut into $1 \mathrm{~mm}^{3}$ cubes and washed several times in $0.1 \mathrm{M}$ phosphate buffer ( $\mathrm{pH} 7.4$ ). All pieces were then incubated for $3 \mathrm{hr}$ in $10 \%$ normal goat serum, washed overnight, and then incubated for $6 \mathrm{hr}$ with the monoclonal antibodies diluted 1:50 in 10\% normal goat serum prepared in $0.1 \mathrm{M}$ phosphate buffer. The muscle was washed repeatedly and then incubated for $6 \mathrm{hr}$ in 1:25 goat anti-mouse biotin-avidin peroxidase diluted in $10 \%$ normal goat serum. After extensive washing, the tissue blocks were osmicated, dehydrated in ethanol, and embedded in Epon. Stained thin sections were examined in a Zeiss EM 10 electron microscope. Motor endplates were localized by the method of Engel et al. (1977).

\section{Results}

The characterization of the anti-sodium channel monoclonal antibodies that were used in this study has been presented in previous reports (Casadei et al., 1984; Casadei and Barchi, 1987). Five monoclonal antibodies were chosen for detailed immunocytochemical study; their properties are given in Table 1. As reported elsewhere, each of these antibodies reacted on immunoblots of whole muscle glycoprotein fractions with the large subunit of the sodium channel resolved in our gel system as a diffuse band at $260 \mathrm{kDa}$. None reacted with the small subunits of the mammalian skeletal muscle sodium channel ( 37 and 38 $\mathrm{kDa}$ proteins). On the basis of competitive binding measurements, 3 of the antibodies (G/B10, L/D3, and A/B2) recognize independent epitopes on the channel, while the remaining $2(\mathrm{~F} /$ E4, I/E3) competetively interact with A/B2 and may share with it a common or closely related epitope.

The immunocytochemical reactivity pattern of these antibodies was first surveyed at the light microscopic level in adult rat skeletal muscle. In fresh-frozen sections of rat anterior tibial muscle, all 5 antibodies exhibited immunofluorescent staining of the muscle surface membrane, although they varied in their relative staining intensity (Fig. 1). Serial cross sections of this mixed fiber type muscle were processed for histochemistry, allowing identification of the muscle fiber types in each section.

Table 1. Properties of monoclonal antibodies

\begin{tabular}{lllll}
$\begin{array}{l}\text { Monoclonal } \\
\text { antibody }\end{array}$ & RIA $^{a}$ & $\begin{array}{l}\text { Immuno- } \\
\text { PPT }^{b}\end{array}$ & $\begin{array}{l}\text { Western } \\
\text { blot }^{c} \\
(\mathrm{kDa})\end{array}$ & Class \\
\hline GB10 & + & + & 260 & $\mathrm{IgG}_{1}$ \\
LD3 & + & - & 260 & $\mathrm{IgG}_{1}$ \\
AB2 & + & + & 260 & $\mathrm{IgG}_{1}$ \\
FE4 & + & + & 260 & $\mathrm{IgG}_{1}$ \\
IE3 & + & + & 260 & $\mathrm{IgG}_{1}$ \\
\hline
\end{tabular}

Data summarized from Casadei et al. $(1984,1986)$ and Casadei and Barchi $(1987)$. ${ }^{a}$ Reactivity with purified sodium channel protein in a solid-phase radioimmunoassay.

${ }^{b}$ Capacity to immunoprecipitate high-affinity ${ }^{3} \mathrm{H}-\mathrm{STX}$ binding from crude solubilized muscle membrane proteins.

' Primary specific immunoreactive component on Western blot of muscle sarcolemmal proteins. 

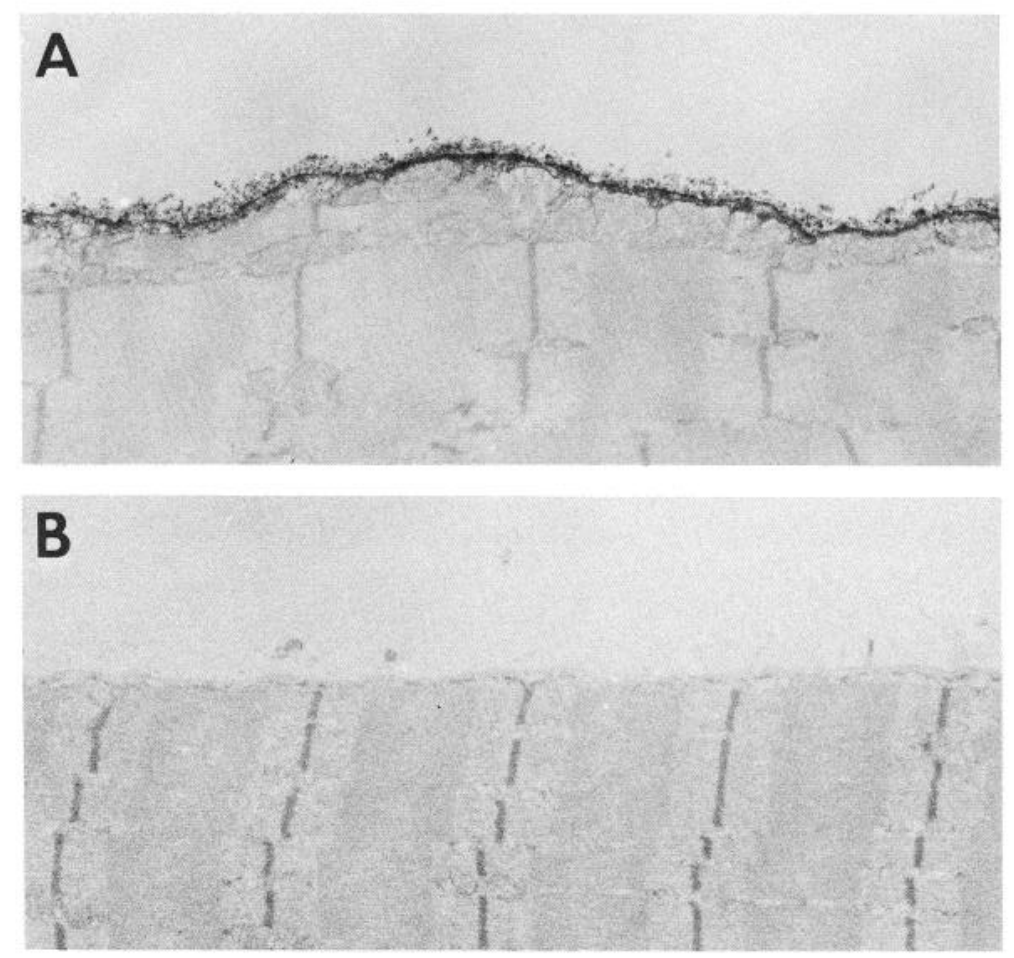

Figure 3. Electron micrographs of anterior tibial muscle sections exposed to monoclonal antibody GB10. The surface membrane was uniformly stained by the antibody $(A)$, although no reaction product was seen in parallel sections incubated with the control immunoglobulin in Ag-8 $(B)$. Very intense reactivity was detected at endplates, where the antibody staining could be traced throughout the secondary folds of the postsynaptic membrane $(C)$. Original magnifications: $A, 6600 ; B$, $7200 ; C, 18,000$.

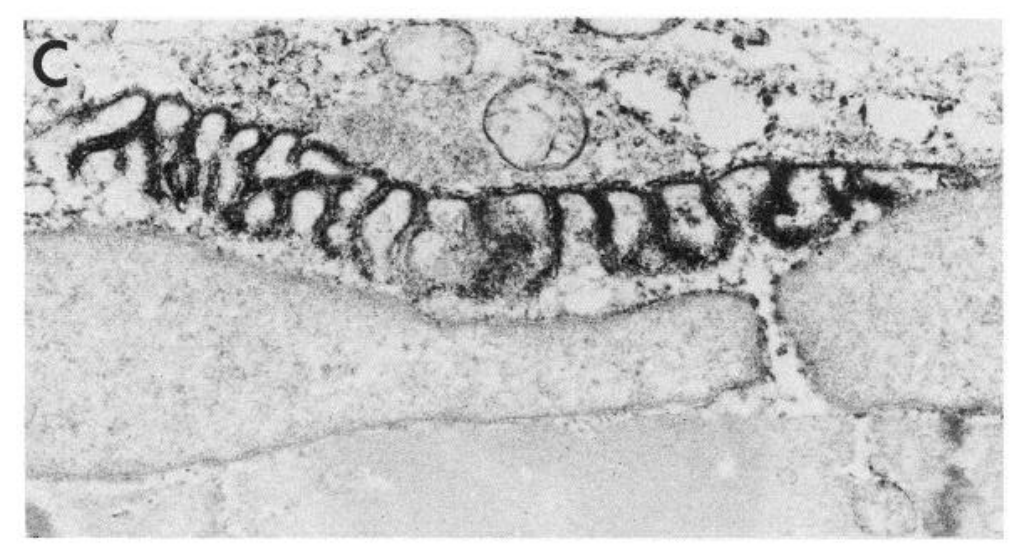

No systematic difference was observed in the reactivity of these antibodies with the sarcolemma of slow- and fast-twitch muscle fibers. We have previously reported comparable results for a polyclonal antiserum raised against the purified rat skeletal muscle sodium channel (Haimovich et al., 1984).

In all the cross and longitudinal sections studied, the surface staining seemed to be homogeneous with the exception of occasional widely scattered very bright stretches averaging $10-15$ $\mu \mathrm{m}$ in length. Unlike the fine line of fluorescence characteristic of the surface staining, these areas were much wider, expanding somewhat into the fiber interior. We examined the possibility that these areas represented endplates using double-labeled preparations exposed simultaneously to fluorescein-conjugated second antibody and rhodamine-conjugated $\alpha$-bungarotoxin. In all the double-stained sections examined, the discrete areas brightly labeled by the sodium channel antibodies coincided with those labeled with $\alpha$-bungarotoxin (Fig. 2).

At the light microscopic level, the labeling intensity of the extrajunctional membrane with antichannel antibodies seemed fairly uniform (Fig. 1). Distinctive areas of patching or regions of high staining intensity were not obvious, although variations in epitope concentration of 2- to 3-fold would probably not be resolved reliably with this approach. This impression was confirmed at the electron microscopic level, where again no major lateral variations in reaction product density were obvious in the extrajunctional membrane (Fig. $3 A$ ). In the postsynaptic membrane, however, the intensity of the staining was clearly greater than that in the surrounding extrajunctional region, indicating a significantly higher density of sodium channels in this region (Fig. 3C). Furthermore, the distribution of label was continuous from the junctional surface to the depths of the secondary synaptic folds, in contradistinction to the preferential distribution of ACh receptors, which are found at the tops of the secondary folds (Salpeter and Harris, 1983). At the electron microscopic level, it is clear that antibodies that label the muscle sodium channel in the postjunctional membrane do not rec- 

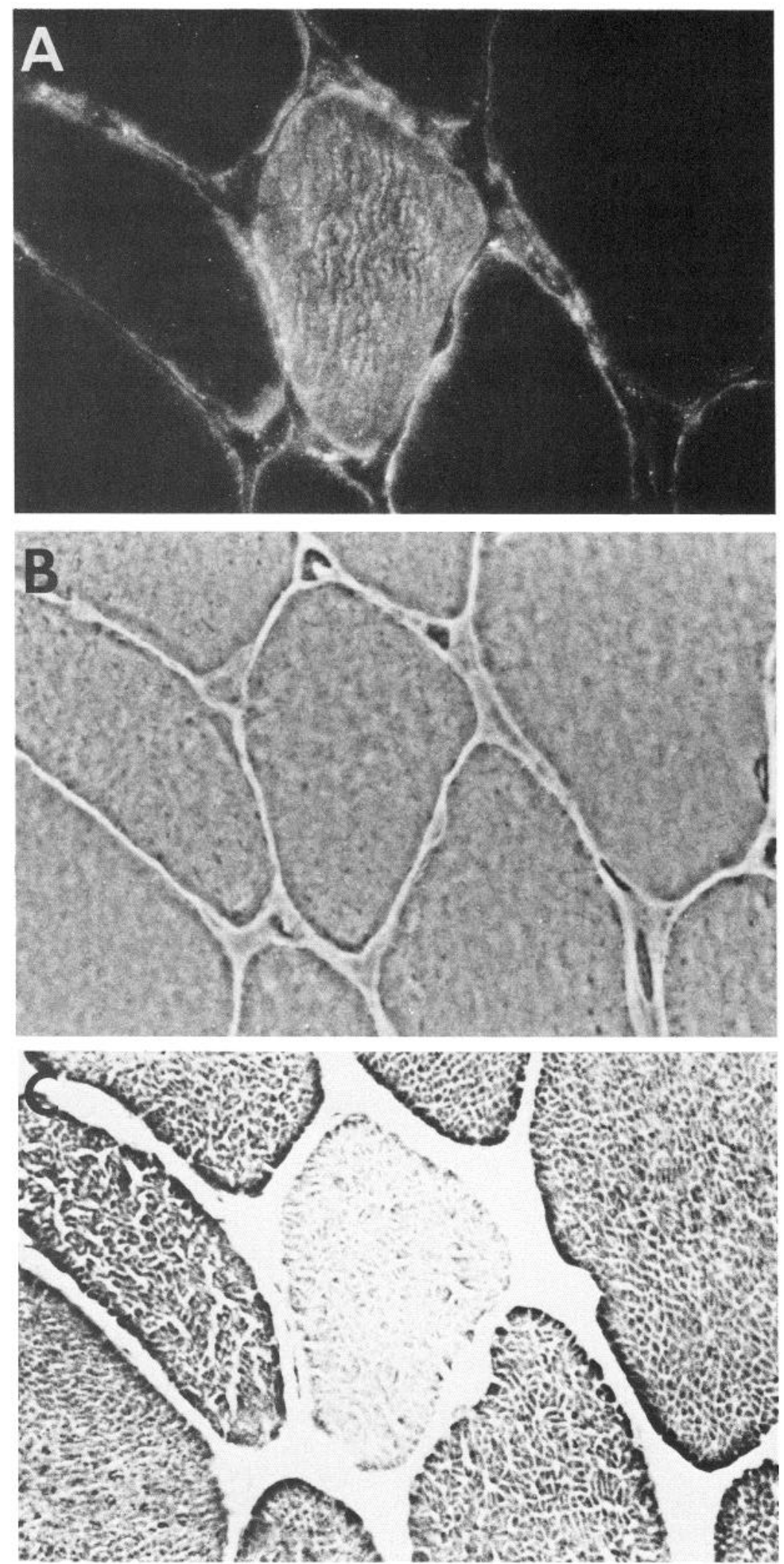

Figure 4. Monoclonal antibodies AB2, FE4, and IE3 produced immunospecific labeling of internal elements in scattered fibers in anterior tibial muscle. In this illustration, frozen serial muscle sections of rat anterior tibial muscle were exposed to 1:50 dilution of the monoclonal antibody $\mathrm{AB} 2$ or processed for ATPase histochemistry. AB2 stained the surface membrane as well as the interior of a limited number of muscle fibers $(A)$, identified as slow-twitch fibers in an ATPase assay $(C)$. A phase-contrast micrograph of the same field is shown in $B$. Original magnification, $\times 600$. ognize the neuronal sodium channel in the adjacent presynaptic membrane. This observation is consistent with the report of Wollner and Catterall (1985), which also documented antigenic differences between the neuronal and muscle sodium channels.
Monoclonal antibodies A/B2, F/E4, and I/E3 demonstrated an additional component of immunoreactivity in both cross and longitudinal sections of anterior tibial muscle. As with the other antibodies, these monoclonals labeled the surface membrane of 

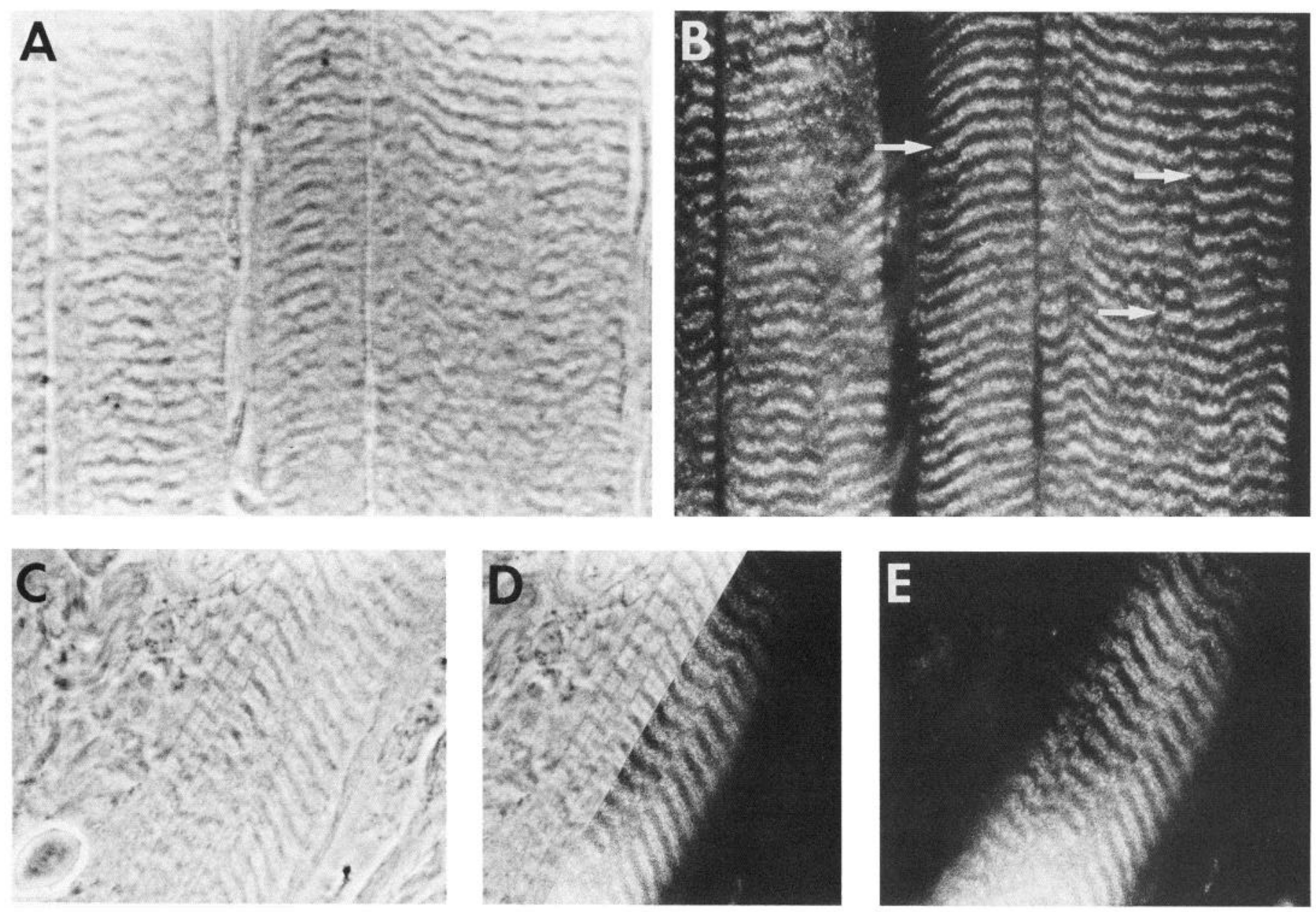

Figure 5. Reactivity of monoclonal antibody $\mathrm{AB} 2$ with longitudinal sections of soleus muscle. $A$ and $B$, The immunofluorescent staining could be localized to repeated transversely oriented bands whose general periodicity corresponded to that of the sarcomeres visible in the same fibers with phase-contrast optics. Arrows in $B$ indicate a number of bands that can be resolved as 2 fluorescent lines separated by a distinct unlabeled region. A superimposed image $(D)$ of phase-contrast $(C)$ and fluorescent photomicrographs $(E)$ of the same section revealed localization of the fluorescent bands to the junction of $\mathrm{A}$ and I bands of each sarcomere $(D)$. Original magnification, $\times 600$.

all fibers in this muscle. In addition, however, these antibodies stained the interior of a limited number of fibers distributed randomly throughout these sections (Fig. $4 A$ ). Using serial cross sections processed for histochemistry, these internally labeled fibers were identified as slow-twitch fibers on the basis of their myofibrillar ATPase cytochemical reactivity (Fig. 4C). The muscle-type specificity of this group was further pursued using the antibody A/B2 in cross and longitudinal sections of rat soleus muscle, which is composed predominantly of slow-twitch fibers. In this muscle, virtually all of the fibers showed bright internal staining. Neither G/B10 nor L/D3 demonstrated consistant staining inside slow or fast muscle fibers.

In cross sections of soleus muscle examined at the light microscopic level, A/B2 clearly localized to structures organized in a repeating pattern within the labeled muscle fibers (Fig. 4). The regular nature of this labeling pattern was more clearly evident in longitudinal sections of soleus muscle fibers (Fig. 5). Here, transversely oriented, regularly spaced bands of fluorescence were seen that could be resolved as 2 fluorescent lines separated by a thin unlabeled region. Closer examination of the superimposed fluorescence and phase-contrast images of the same field showed that the fluorescence doublets were localized at the junction of A and I bands in each sarcomere, suggesting that $\mathrm{A} / \mathrm{B} 2$ reacted with sodium channels in the $\mathrm{T}$-tubular membranes known to be situated at these levels in mammalian muscle (Ishikawa, 1983).

In order to evaluate the possibility that $\mathrm{A} / \mathrm{B} 2$ cross-reacted with components of the contractile apparatus, we examined the reactivity of $\mathrm{A} / \mathrm{B} 2$ with crude preparations of actomyosin proteins from rat soleus and extensor digitorum longus muscle. These fractions have been shown by others to contain myosin heavy and light chains, actin, tropomyosin, and C-troponin (Gambke and Rubinstein, 1984). Our antibody did not react with any of these proteins either on immunoblots or in a solidphase radioimmunoassay. Similarly, no reactivity was detected with high concentrations of purified rabbit tropomyosin, troponin, or actin on immunoblots, although A/B2 cross-reacted with rabbit muscle and the pattern of staining and muscle type specificity seen immunocytochemically in rabbit were indistinguishable from those presented here for rat muscle.

The location of this internal epitope recognized by $\mathrm{A} / \mathrm{B} 2$ was then evaluated at the electron microscopic level. Bundles of 

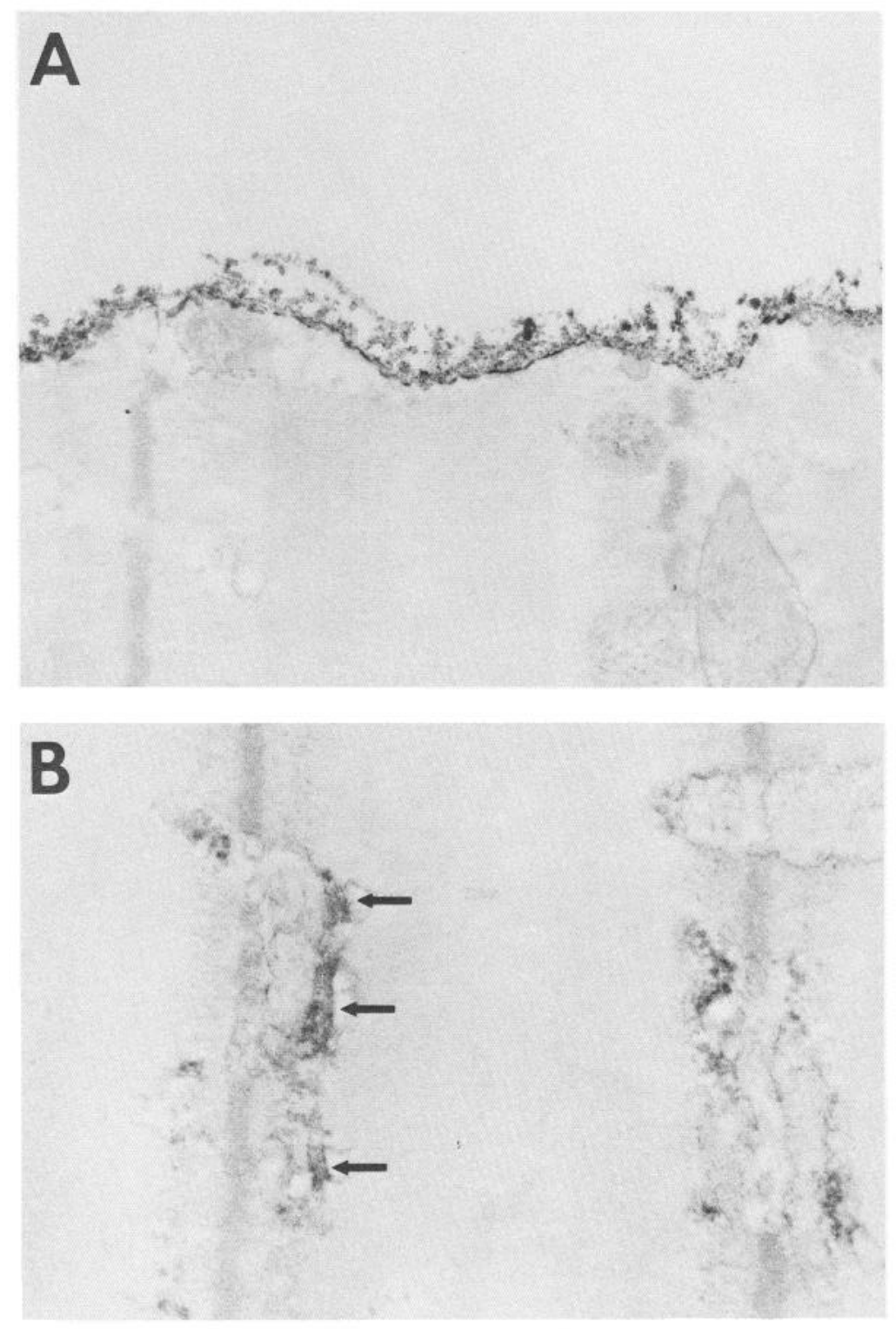

Figure 6. Electron micrographs of soleus muscle fibers exposed to monoclonal AB2. $A$, Reactivity of unpermeabilized muscle fibers is restricted to the surface membrane. $B$, After permeabilization with $0.05 \%$ saponin, $A B 2$ also reacted with elements of the T-tubular system (arrows), although no reactivity was seen with membranes of the adjacent sarcoplasmic reticulum or with contractile proteins. Original magnifications: $A, 25,000 ; B, 30,000$. soleus fibers were incubated with the antibody either with or without permeabilization of their membranes by exposure to $0.05 \%$ saponin. Fibers not exposed to saponin revealed only surface staining without any intracellular reactivity (Fig. 6A). After treatment with saponin, however, reaction product could be seen both along the sarcolemma and in association with internal membrane elements (Fig. 6B). This internal staining was confined completely to the T-tubular membranes; no labeling was seen of immediately adjacent elements of the sarcoplasmic reticulum or of any components of the contractile apparatus.

The reactivity of these 2 groups of antibodies was also surveyed at the light microscopic level with rat cardiac ventricular fibers. None of these monoclonals reacted with the surface membrane of these cardiac fibers. A/B2, representing the second group of monoclonal antibodies, reacted strongly in a transversely oriented pattern within the cardiac fibers (Fig. 7). When compared with the pattern seen in soleus muscle, the bands produced by A/B2 were wider, appeared somewhat brighter, and were never resolved as doublets.

\section{Discussion}

Recent electrophysiological studies have suggested that sodium channels in muscle may not be uniformly distributed along the fiber surface (Almers et al., 1983; Beam et al., 1985), and binding measurements with various neurotoxins have raised the possibility that different subpopulations of sodium channels may be present even in adult muscle fibers (Jaimovich et al., 1982, 1983; Barhanin et al., 1984; Cruz et al., 1985). Unfortunately, the cytochemical localization of sodium channels at the light and electron microscopic level in skeletal muscle has presented a challenge in the past because of the lack of suitable markers. The availability of a panel of monoclonal antibodies specific for the rat skeletal muscle sodium channel $\alpha$-subunit has now allowed us to pursue these issues directly with immunocytochemical techniques. 

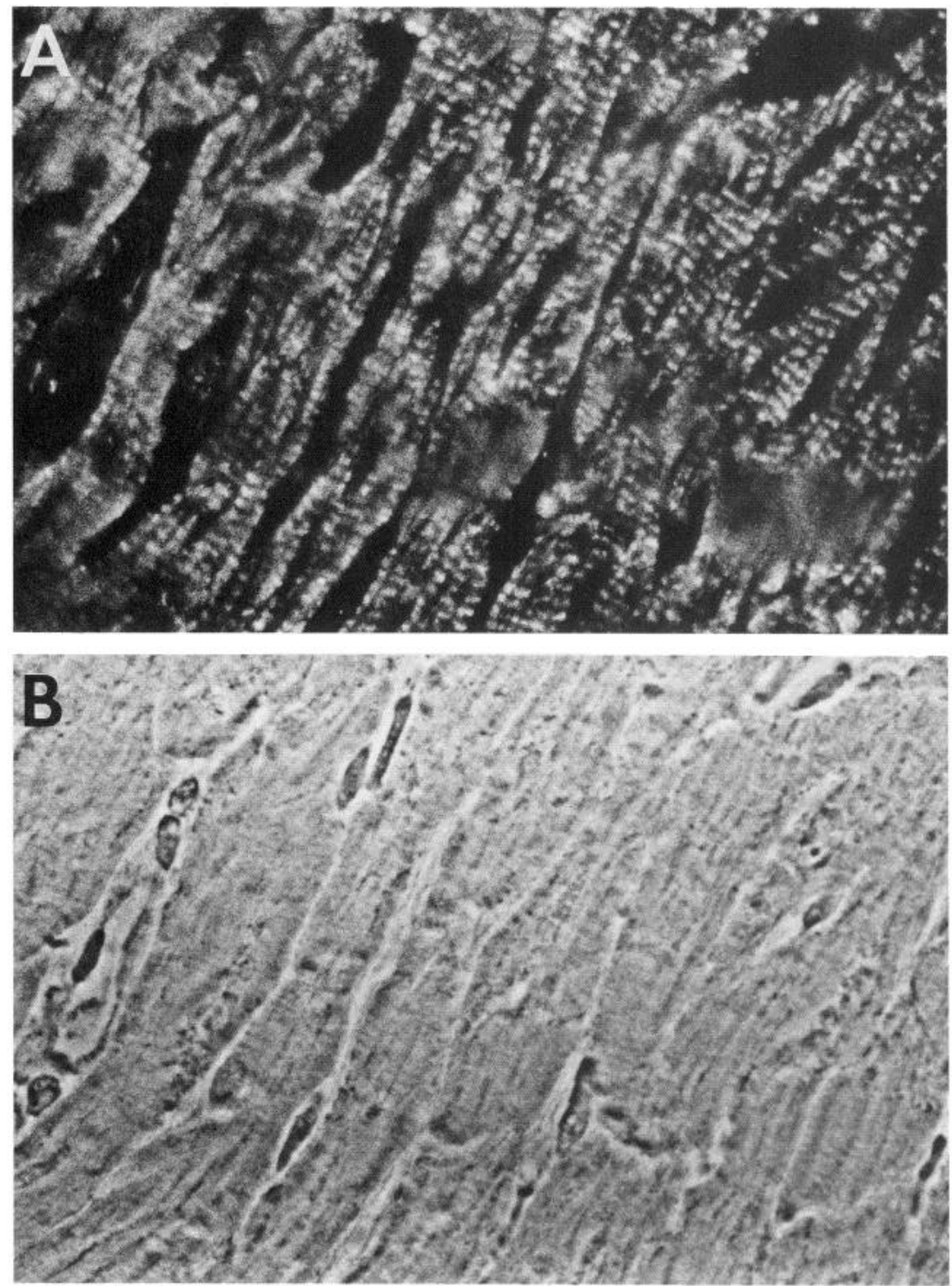

Figure 7. Monoclonal antibody AB2 also labeled rat cardiac ventricular fibers in a pattern consistent with the distribution of T-tubular elements in this tissue. $A$, Longitudinal sections of ventricular fibers exposed to 1:50 dilution of AB2. B, Matched phase-contrast image. Original magnification, $\times 600$.

Of 29 anti-channel monoclonal antibodies in our panel (Casadei and Barchi, 1987), only 5 monoclonals produced sufficient immunocytochemical reactivity with frozen sections of adult rat skeletal muscle to be useful for such studies. At the light microscopic level, all of our probes exhibited the common feature of staining at the muscle fiber surface membrane. Examination of cross and longitudinal sections of mixed fiber type muscle, such as anterior tibial, allowed an accurate comparison of the extent of reactivity with both slow and fast fibers; all the antibodies seemed to react equally with the sarcolemma of both muscle types.

Using loose patch-recording techniques, Almers et al. (1983) have previously documented variations of up to 3 -fold in $\mathrm{Na}^{+}$ current density over distances of 10-30 $\mu \mathrm{m}$ along the surface of frog sartorius muscle fibers. Recently, Beam et al. (1985) reported 2-fold variations in $\mathrm{Na}^{+}$current density between spots spaced $10 \mu \mathrm{m}$ apart on the extrajunctional surface of snake skeletal muscle fibers. In our preparations, the surface staining of both fast and slow muscle fibers appeared homogenous at the light microscopic level, although very small patches of less than $1 \mu \mathrm{m}$ were occasionally seen that were most likely the result of artifacts caused during the processing of the muscle. The apparent discrepancy between our results and those reported previously using electrophysiological techniques may reflect differences in assay sensitivity; a 2 -fold or less variation in the level of immunofluorescence would be difficult to differentiate in our sections from normal experimental variability. Another possibility is that sodium channels are more homogeneously distributed on the surface of mammalian than on nonmammalian muscle. Alternatively, sodium channels might be evenly distributed in all cases but show regional variability in their availability for activation, thus giving rise to the gradations in sodium current measured with the loose patch electrode. Finally, it is possible that some surface channels are not immunoreactive but are detected with electrophysiological techniques.

Immunofluorescence at the light microscopic level clearly shows higher densities of sodium channel immunoreactivity in the endplate region of rat muscle fibers as compared with the surrounding extrajunctional membrane. This is consistent with electrophysiological measurements suggesting the existence of sodium channels in the postsynaptic region (Nastuk and Alexander, 1973; Thesleff et al., 1974), and especially with the work 
of Beam et al. (1985) indicating that the $\mathrm{Na}^{+}$current density in the immediate vicinity of the snake abdominal muscle endplate may be 10 -fold higher than that in the extrasynaptic region. We would expect density differences of this magnitude to be detectable with immunofluorescence techniques, and they are clearly seen with our antibodies. Using fluorescently labeled neurotoxins to tag sodium channels, Angelides (1986) also detected immobilized sodium channels localized at the neuromuscular junction of innervated muscle at the light microscopy level.

The distribution of sodium channels at the neuromuscular junction differs from that previously described for the ACh receptor. Unlike the ACh receptors, which are located only on the top of the postjunctional folds (Salpeter and Harris, 1983), sodium channel immunoreactivity at the electron microscopic level is detected throughout the secondary folds of the postsynaptic region.

More surprising were the variations in immunoreactivity patterns demonstrated by our antibodies between fiber types in adult skeletal muscle. Although all antibodies labeled both slowand fast-fiber surface membranes, 3 antibodies also consistently stained the T-tubular membranes of slow-twitch fibers. These antibodies, however, did not recognize sodium channels in the $\mathrm{T}$-tubular membranes of fast-twitch fibers.

The immunoreactivity of these antibodies in cardiac muscle, with a wider repeating transverse band that did not demonstrate a doublet pattern, is also consistent with T-tubular labeling based on the larger area of the cardiac $T$-tubular system and its known localization at the Z-line rather than at the A-I junctions (Simpson and Oerlelis, 1962; Lullman and Peters, 1977).

Our results indicate that there must be at least 3 subpopulations of sodium channels present in adult skeletal muscle, which differ in one or more epitopes recognized by these antibodies: channels in the sarcolemma of fast and slow fibers, those in slow fiber T-tubules, and those in the T-system of fast fibers. These channels may all share the same primary sequence and be the product of the same gene, differing only in their posttranslational modification such as their degree of glycosylation. Other studies from our laboratory, however, suggest that the epitope recognized by $\mathrm{A} / \mathrm{B} 2$ that differentiates fast from slow T-system channels is not a carbohydrate residue (Casadei and Barchi, 1987). The subtypes could represent the expression of different, although closely related, channel messages. In rat brain, for example, 3 related sodium channel messages have been identified; in the 2 for which complete sequence information is available, their homology exceeds $90 \%$ (Noda et al., 1986). Further work will be needed to differentiate between these possibilities.

\section{References}

Agnew, W. S. (1984) Voltage-regulated sodium channel molecules. Annu. Rev. Physiol. 46: 517-530.

Almers, W., P. R. Stanfield, and W. Stuhmer (1983) Lateral distribution of sodium and potassium channels in frog skeletal muscle: Measurements with a patch-clamp technique. J. Physiol. 336: 261284.

Angelides, K. (1986) Fluorcscently labelled $\mathrm{Na}^{+}$channels are localized and immobilized to synapses of innervated muscle fibers. Nature 321 : 63-66.

Baer, M., P. M. Best, and H. Reuter (1976) Voltage-dependent action of tetrodotoxin in mammalian cardiac muscle. Nature 263: 344-345.

Barchi, R. L. (1984) Voltage-sensitive sodium channels. Molecular properties and functional reconstitiution. Trends Biochem. Sci. 9: 358-361.

Barhanin, J., M. Ildefonse, O. Rougier, S. V. Sampaio, J. R. Giglio, and
M. Lazdunski (1984) Tityus gamma toxin, a high affinity effector of $\mathrm{Na}^{+}$channel in muscle, with a selectivity for channels in the surface membrane. Pfluegers Arch. 400: 22-27.

Beam, K. G., J. H. Caldwell, and D. T. Campbell (1985) Na channels in skeletal muscle concentrated near the neuromuscular junction. Nature 313: 588-590.

Brooke, M., and K. Kaiser (1969) Some comments on the histochemical characterization of muscle adenosine triphosphatase. J. Histochem. Cytochem. 17: 431-432.

Casadei, J. M. and R. L. Barchi (1987) Monoclonal antibodies against the voltage-sensitive sodium channel from rat skeletal muscle: Mapping antibody binding sites. J. Neurochem. 48: 773-778.

Casadei, J. M., R. D. Gordon, L. A. Lampson, D. L. Schotland, and R. L. Barchi (1984) Monoclonal antibodies against the voltage-sensitive $\mathrm{Na}^{+}$channel from mammalian skeletal muscle. Proc. Natl. Acad. Sci. USA 81: 6227-6231.

Casadei, J. M., R. D. Gordon, and R. L. Barchi (1986) Immunoaffinity isolation of $\mathrm{Na}^{+}$channels from rat skeletal muscle: Analysis of subunits. J. Biol. Chem. 261: 4328-4333.

Catterall, W. A. (1984) The molecular basis of neuronal exitability. Science 223: 653-661.

Chicheportiche, R., M. Balerna, A. Lombet, G. Romey, and M. Lazdunski (1980) Synthesis of new, highly radioactive tetrodotoxin derivatives and their binding properties to sodium channels. Eur. J. Biochem. 104: 617-625.

Cohen, C. J., B. P. Beam, T. J. Colatsky, and R. W. Tsien (1981) Tetrodotoxin block of sodium channels in rabbit Purkinje fibers: Interactions between toxin binding and channel gating. J. Gen. Physiol. 78: 383-411.

Constantin, L. L., and S. R. Taylor (1971) Active and passive shortening in voltage-clamped frog muscle fibers. J. Physiol. (Lond.) 218 : 13-15P.

Cruz, L. J., W. R. Gray, B. M. Olivera, R. D. Zeikus, L. Kerr, D. Yoshikami, and E. Moczydlowski (1985) Conus geographus toxins that discriminate between neuronal and muscle sodium channels. J. Biol. Chem. 260: 9280-9288.

Engel, A. G., J. M. Lindstrom, E. H. Lambert, and V. A. Lennon (1977) Ultrastructural localization of the acetylcholine receptor in myasthenia gravis and in its experimental autoimmune model. Neurology 27: 307-312

Gambke, B., and N. A. Rubinstein (1984) A monoclonal antibody to the embryonic myosin heavy chain of rat skeletal muscle. J. Biol. Chen. 259: 12092-12100.

Haimovich, B., E. Bonilla, J. Casadei, and R. Barchi (1984) Immunocytochemical localization of the mammalian voltage-dependent sodium channels using polyclonal antibodies against the purified protein. J. Neurosci. 4: 2259-2268.

Harris, J. B., and S. Thesleff (1971) Studies on tetrodotoxin resistant action potentials in denervated skeletal muscle. Acta Physiol. Scand 83: $382-388$

Ishikawa, H. (1983) Fine structure of skeletal muscle. In Cell and Muscle Motility, Vol. 4., R. W. Dowben and J. W. Shay, eds., pp. 184, Plenum, New York.

Jaimovich, E., M. Ildefonse, J. Barhanin, O. Rougier, and M. Lazdunski (1982) Centuroides toxin, a selective blocker of surface $\mathrm{Na}^{+}$channels in skeletal muscle: Voltage-clamp analysis and biochemical characterization of the receptor. Proc. Natl. Acad. Sci. USA 79: 3896-3900.

Jaimovich, E., R. Chicheportiche, A. Lombet, M. Lazdunski, M. Ildefonse, and O. Rougier (1983) Differences in the properties of $\mathrm{Na}^{+}$ channels in muscle surface and t-tubular membranes revealed by tetrodotoxin derivatives. Pluegers Arch. 397: 1-5.

Lullmann, $H$., and T. Peters (1977) Plasmalemmal calcium in cardiac excitation-contraction coupling. Clin. Exp. Pharmacol. Physiol. 4: 49-57.

Moczydlowski, E. G., B. M. Olivera, W. R. Gray, and G. R. Strichartz (1986) Discrimination of muscle and neuronal sodium channel subtypes by binding competition between ${ }^{3} \mathrm{H}$-saxitoxin and $\mu$-conotoxins. Proc. Natl. Acad. Sci. USA 83: 5321-5325.

Nastuk, W. L., and J. T. Alexander (1973) Non-homogenous electrical activity in single muscle fibers. Fed. Proc. 32: 333.

Noda, M., I. Takayuki, T. Kayano, H. Suzuki, II. Takeshima, M. Kurasaki, H. Takahashi, and S. Numa (1986) Existence of distinct sodium channel messenger RNAs in rat brain. Nature 320: 188-192.

Padykula, H., and E. Herman (1955) The specificity of the histochemical method for adenosine triphosphatase. J. Histochem. Cytochem. 3: $170-195$. 
Pappone, P. (1980) Voltage-clamp experiments in normal and denervated mammalian skeletal muscle fibers. J. Physiol. (Lond.) 305: 377410.

Ritchie, J. M., and R. Rogart (1977) The binding of saxitoxin and tetrodotoxin to excitable tissues. Rev. Physiol. Biochem. Pharmacol. 79: $1-50$.

Salpeter, M. M., and R. Harris (1983) Distribution and turnover rate of receptors throughout the junction folds at a vertebrate neuromuscular junction. J. Cell Biol. 96: 1781-1785.
Simpson, F. O., and S. J. Oertelis (1962) The fine structure of sheep myocardial cells: Sarcolemmal invagination and the transverse tubular system. J. Cell Biol. 12: 91-100.

Thesleff, S., F. Vyskogil, and M. R. Ward (1974) The action potential in end-plate and extrajunctional regions of rat skeletal muscle. Acta Physiol. Scand. 91: 196-202.

Wollner, D. A., and W. A. Catterall (1985) Antigenic differences among the voltage-sensitive sodium channels in the peripheral and central nervous systems and skeletal muscle. Brain Res. 331: 145-149. 